\title{
EFEITO DE DOSES DE TORTA DE FILTRO E MODO DE APLICAÇÃO SOBRE A PRODUTIVIDADE E QUALIDADE TECNOLÓGICA DA SOQUEIRA DE CANA-DE-AÇÚCAR
}

\author{
Effect of filter cake doses and methol of application on yield and \\ technologycal quality of sugar cane ratoon \\ Paulo Roberto Fávero de Fravet ${ }^{1}$, Rogério Augusto Brem Soares², \\ Regina Maria Quintão Lana ${ }^{3}$, Ângela Maria Quintão Lana ${ }^{4}$, Gaspar Henrique Korndörfer ${ }^{5}$
}

\begin{abstract}
RESUMO
A expansão do setor sucroalcooleiro aumenta áreas de plantio, assim como volumes de resíduos (como a torta de filtro) que podem ser utilizados na agricultura como fonte de nutrientes, reduzindo a contaminação ambiental e os custos com adubação, entretanto, faltam informações sobre modos de aplicação. Nesse contexto, conduziu-se este trabalho, com o objetivo de avaliar doses de torta de filtro e modos de aplicação (superficial na linha e incorporado na entrelinha) sobre as variáveis tecnológicas e produtividade da cana soca. O experimento foi instalado em soqueira de cana-de-açúcar (SP 81-3250), cultivada em solo de textura argilosa, localizado no município de Goianésia/GO. O delineamento experimental utilizado foi em blocos ao acaso (DBC), com onze tratamentos e 5 repetições, em esquema fatorial $5 \times 2+1$ que correspondem a 5 doses crescentes de torta de filtro $\left(0,10,20,40\right.$ e $\left.^{2} 0 \mathrm{t} \mathrm{ha}^{-1}\right)$, combinadas a 2 modos de aplicação (na linha superficial e na entre linha incorporada) e um tratamento adicional correspondente à adubação mineral da Usina Jalles Machado S.A.. A aplicação de torta de filtro na cana-soca na dose de 70 t ha $^{-1}$ proporcionou a maior produção de colmos de cana-de-açúcar, independentemente do modo de aplicação.
\end{abstract}

Termos para indexação: Resíduos, adubação, cana-soca, métodos de preparo, Saccharum officinarum.

\begin{abstract}
The expansion of sugarcane (Saccharum officinarum L.) cultivation for the production of sugar or ethanol has increased cultivated land as well as the volume of residues (such as filter cake) that may be used in agriculture as a nutrient source, reducing environmental contamination and fertilization costs. However, there is a lack of information about the best way of application. In this way, the objective of the present work was to evaluate the effect of the filter cake dose and the application method (on the ground in the row and incorporated between rows) on the technological variables and ratoon yield. The experiment was installed on a sugar cane ratoon (SP 81-3250), cultivated in clay soil in the municipality of Goianésia, State of Goiás, Brazil. The experimental delineation used was randomized blocks with eleven treatments and five replications, in a factorial scheme 5 X $2+1$, which correspond to 5 increasing doses of filter cake $\left(0,10,20,40 \mathrm{e} 80 \mathrm{t} \mathrm{ha}^{-1}\right)$ with 2 modes of application (over the ground in the row and incorporated in the between row) and an additional treatment corresponding to the mineral nutrition done by the company Usina Jalles Machado S.A.. The highest sugar cane ratoon production was obtained when $70 \mathrm{tha}^{-1}$ of filter cake were used, independently of the mode of application.
\end{abstract}

Index terms: Residue, fertilization, sugarcane ratoon, soil till, Saccharum officinarum.

(Recebido em 4 de março de 2009 e aprovado em 30 de setembro de 2009)

\section{INTRODUÇÃO}

O Brasil é o maior produtor mundial de cana-deaçúcar (Saccharum officinarum L.) com participação em $10 \%$ do total da área destinada à agricultura (Alvarenga \& Queiroz, 2008). Além de ser o maior produtor e exportador mundial de açúcar refinado, também é consumidor e exportador do álcool utilizado como combustível de veículos. A expansão do mercado de álcool e açúcar leva o setor sucroalcooleiro a projetar aumento na produção de cana-de-açúcar, que deverá atingir 580 milhões de toneladas de cana-de-açúcar na safra 2010/2011 (Produção..., 2006).

Para atender a essa expansão, mais áreas são plantadas e, consequentemente maiores volumes de resíduos (como a torta de filtro) são gerados. Estes podem ser utilizados na agricultura como fonte de nutrientes, reduzindo a contaminação ambiental e os custos com

\footnotetext{
${ }^{1}$ Universidade Federal de Uberlândia/UFU - Uberlândia, MG

'Usina Jalles Machado - Goianésia, GO

${ }^{3}$ Instituto de Ciências Agrárias, Universidade Federal de Uberlândia/UFU - Av. Amazonas, s/n, Bloco 4C, Campus Umuarama - Cx. P. 593 - $38.400-734$ rmqlana@iciag.ufu.br

${ }^{4}$ Universidade Federal de Minas Gerias/UFMG - Departamento de Zootecnia, EV - Belo Horizonte, MG

${ }^{5}$ Instituto de Ciências Agrárias, Universidade Federal de Uberlândia/UFU - Uberlândia, MG
} 
adubação. Segundo a UDOP - União dos Produtores de Bioenergia (2007), citado por Alvarenga \& Queiroz (2008), a torta de filtro e a vinhaça podem substituir adubos químicos e acarretar uma diminuição dos custos em torno de US\$ 60 por hectare. Cada tonelada de cana moída gera em torno de $40 \mathrm{~kg}$ de torta de filtro (Korndörfer, 2003) que é resultante da mistura do processo de clarificação do açúcar (lodo de decantação) com o bagaço moído (Alvarenga \& Queiroz, 2008). É viável a substituição da adubação química pela orgânica sem perdas na qualidade da matéria-prima e nos rendimentos de colmos e de açúcar mascavo artesanal (Anjos et al., 2007).

Por muitos anos, a adubação da cana-de-açúcar garantiu o fósforo apenas no plantio, acreditando-se que a cana-soca não respondia à adubação com este nutriente (Bittencourt et al., 2006). De acordo com Zambello Junior et al. (1980), o fósforo provoca aumentos significativos na produção de soqueiras de cana-de-açúcar. Segundo Medeiros (1998), o uso de doses elevadas de fósforo no plantio pode ser insuficiente necessitando-se suprir as soqueiras, tendo assim, aumentos significativos de produção. Barbosa et al. (2002) e Oliveira et al. (2002) verificaram grande extração de fósforo pela planta da cana e que cerca de $80 \%$ desse nutriente está contido nos colmos industrializáveis. Korndörfer (2003) acrescenta que maior o teor de fósforo do solo, reflete no caldo da cana-deaçúcar.

A torta de filtro, composto basicamente orgânico, tem composição química variável e apresenta altos teores de matéria orgânica, fósforo, nitrogênio, cálcio e possui, ainda, teores consideráveis de potássio, magnésio (Nunes Júnior, 2005), e expressivas quantidades de Fe, Mn, Zn e $\mathrm{Cu}$ (Cerri et al., 1988). Uma dose de $20 \mathrm{t} \mathrm{ha}^{-1}$ de torta de filtro na base úmida ou $5 \mathrm{t} \mathrm{ha}^{-1}$ na base seca (M.S.) pode fornecer $100 \%$ do nitrogênio, $50 \%$ de fósforo, $15 \%$ de potássio, $100 \%$ de cálcio e $50 \%$ de magnésio (Nunes Júnior, 2005) e pode ser aplicada em área total em pré-plantio, no sulco ou nas entrelinhas de plantio. Nunes Júnior (1999) acrescenta que não é necessário incorporar a torta de filtro quando a cana é colhida sem despalha a fogo. Entretanto, existem poucos estudos sobre a quantidade e forma de aplicação desse resíduo em cana-soca (Medeiros et al., 2003).

A localização do adubo no solo é importante para a cultura da cana, principalmente para o fósforo, em razão da sua pouca mobilidade no solo. Dessa forma, admitese que, aumentando o volume de solo fertilizado ao redor das raízes, ocorrerá maior contato nutriente-raiz e, portanto, um aumento na absorção do nutriente (Sleight et al., 1984).

Nesse contexto, conduziu-se este trabalho, com o objetivo de avaliar a resposta de diferentes doses de torta de filtro e o modo de aplicação (superficial na linha e incorporado na entrelinha) sobre as variáveis tecnológicas e produtividade da cana soca.

\section{MATERIAL E MÉTODOS}

O trabalho foi desenvolvido com a cultura da canade-açúcar numa área de sistema plantio convencional e sem irrigação, em Latossolo Vermelho Amarelo (LVA) textura argilosa, situado geograficamente na latitude $15^{\circ} 18^{\prime} \mathrm{S}$, longitude $49^{\circ} 7, \mathrm{~W}$ e altitude de $650 \mathrm{~m}$, localizado na Fazenda Pai João da Usina Jalles Machado S.A., no município de Goianésia/GO, durante as safras 2003 e 2004. $\mathrm{O}$ experimento foi instalado após o terceiro corte do canavial em 2003 e colhido em 2004.

Na Tabela 1, apresenta-se a caracterização química e física do solo de 4 (quatro) subamostras da linha e 1 (uma) subamostra da entrelinha realizada antes da implantação do experimento.

A torta de filtro é composta por resíduos, solúveis e insolúveis, que equivalem ao precipitado (lodo) formado na etapa de clarificação do caldo de cana. O lodo formado, composto orgânico e inorgânico insolubilizado, passa por um processo de filtração a vácuo, recebendo, então, a denominação de torta de filtro. Na Tabela 2, apresentam-se as características físico-químicas da torta de filtro da Usina Jalles Machado S.A.

Tabela 1 - Análise química e física do solo antes da implantação do experimento em 2003.

\begin{tabular}{|c|c|c|c|c|c|c|c|c|c|c|c|c|c|}
\hline $\begin{array}{l}\text { Profundidade } \\
\qquad(\mathrm{cm})\end{array}$ & $\begin{array}{c}\mathrm{pH} \\
\mathrm{H}_{2} \mathrm{O}\end{array}$ & $\begin{array}{c}\mathrm{P} \\
\mathrm{mg} \mathrm{d}\end{array}$ & $\begin{array}{r}\mathrm{K} \\
\mathrm{m}^{-3}\end{array}$ & $\mathrm{Ca}$ & $\mathrm{Mg}$ & $\begin{array}{l}\mathrm{H}+\mathrm{Al} \\
-\mathrm{cmol}_{\mathrm{c}}\end{array}$ & $\begin{array}{l}\text { SB } \\
\mathrm{m}^{-3}--\end{array}$ & $\mathrm{t}$ & $\mathrm{T}$ & $\begin{array}{c}\text { MO } \\
\mathrm{g} \mathrm{dm}^{-3}\end{array}$ & Areia & Silte & Argila \\
\hline $0-20$ & 5,6 & 2,4 & 75,0 & 1,2 & 0,9 & 2,8 & 2,2 & 2,2 & 5,0 & 25,6 & 58,3 & 6,4 & 35,3 \\
\hline $20-40$ & 5,1 & 0,4 & 14,0 & 0,5 & 0,5 & 3,1 & 1,0 & 1,6 & 4,1 & 24,4 & 57,2 & 5,1 & 37,7 \\
\hline
\end{tabular}

P e K: extraídos com solução de $\mathrm{HCl} 0,05 \mathrm{~mol} \mathrm{l}^{-1} \mathrm{e} \mathrm{H}_{2} \mathrm{SO}_{4} 0,025 \mathrm{~mol} \mathrm{l}^{-1}$.

$\mathrm{Ca}, \mathrm{Mg}$ e Al: extraídos com solução de $\mathrm{KCl} 1,0 \mathrm{~mol}^{-1}$.

M.O. = Método Walkley-black. 
Tabela 2 - Composição mineral da matéria seca da torta de filtro da Usina Jalles Machado S.A..

\begin{tabular}{|c|c|c|c|c|c|c|c|c|c|c|c|c|}
\hline $\mathrm{pH}$ & $\begin{array}{c}\text { Relação } \\
\text { C/N }\end{array}$ & $\begin{array}{c}\text { Relação } \\
\text { C/P }\end{array}$ & MO & Umidade & $\mathrm{Ca}$ & $\mathrm{Mg}$ & S & $\begin{array}{l}\mathrm{P}_{2} \mathrm{O}_{5} \\
\left(\mathrm{H}_{2} \mathrm{O}\right)\end{array}$ & $\begin{array}{c}\mathrm{P}_{2} \mathrm{O}_{5} \\
\left(\mathrm{CNA}+\mathrm{H}_{2} \mathrm{O}\right)\end{array}$ & $\begin{array}{c}\mathrm{P}_{2} \mathrm{O}_{5} \text { (Ácido } \\
\text { Cítrico 2\%) }\end{array}$ & $\begin{array}{l}\mathrm{P}_{2} \mathrm{O}_{5} \\
\text { (Total) }\end{array}$ & $\mathrm{K}_{2} \mathrm{O}$ \\
\hline 4,5 & 20,9 & 17,65 & 20,1 & 71,4 & 2,43 & 0,26 & 0,39 & 0,75 & 0,92 & 0,92 & 2,25 & 0,3 \\
\hline
\end{tabular}

O experimento foi conduzido utilizando-se o delineamento experimental de blocos ao acaso (DBC) com 11 (onze) tratamentos e 5 (cinco) repetições, em esquema fatorial $5 \times 2+1$, que correspondem a 5 doses crescentes de torta de filtro com $71,4 \%$ de umidade $(0,10,20,40$ e 80 t ha ${ }^{-1}$ ) combinadas a 2 modos de aplicação (na linha superficial e na entrelinha incorporada) e um tratamento adicional correspondente à adubação mineral da Usina Jalles Machado S.A.. A incorporação foi realizada de forma mecanizada.

Para a adubação mineral da Usina Jalles Machado S.A., aplicou-se $61 \mathrm{~kg} \mathrm{ha}^{-1}$ de amônia anidra, $73 \mathrm{~kg} \mathrm{ha}^{-1}$ de MAP e $198 \mathrm{~kg} \mathrm{ha}^{-1}$ de cloreto de potássio, o que corresponde a $50 \mathrm{~kg} \mathrm{ha}^{-1}$ de $\mathrm{N}, 35 \mathrm{~kg} \mathrm{ha}^{-1}$ de $\mathrm{P}_{2} \mathrm{O}_{5}$ e $120 \mathrm{~kg}$ $\mathrm{ha}^{-1}$ de $\mathrm{K}_{2} \mathrm{O}$.

Na Tabela 3, apresenta-se o fornecimento $\left(\mathrm{kg} \mathrm{ha}^{-1}\right)$ dos nutrientes $\mathrm{N}, \mathrm{P}_{2} \mathrm{O}_{5}, \mathrm{~K}_{2} \mathrm{O}, \mathrm{Ca}, \mathrm{Mg}$, S e da matéria orgânica (M.O.) proporcionada pela aplicação dos tratamentos (Bataglia et al., 1985).

A área das parcelas foi de $140 \mathrm{~m}^{2}$, ou seja, 5 linhas de 20 $\mathrm{m}$ de comprimento, com espaçamento entre as linhas de 1,40 m.

Antes do plantio da cana, aplicou-se na área 1,0 t ha $^{-1}$ de calcário dolomitico (PRNT $\left.=90 \%\right)$ e para a adubação de plantio, $600 \mathrm{~kg} \mathrm{ha}^{-1}$ de $04-28-20+3 \%$ de zinco. A variedade utilizada no experimento foi a SP 81-3250, que é de ciclo médio e exigente em fertilidade do solo. Não foi realizada adubação mineral de cobertura.

As avaliações previstas neste estudo foram: Brix do caldo (\%); Pol da cana (\%); produtividade de colmos em toneladas por hectare $(\mathrm{TCH})$, produtividade de sacarose em toneladas por hectare (TPH).

As amostras provenientes do campo, compostas por colmo da cana-de-açúcar, foram individualmente identificadas e levadas ao laboratório industrial para aferições quantitativas e qualitativas. As amostras foram submetidas às seguintes aferições quantitativas: determinação da produtividade de cana-de-açúcar $\left(\mathrm{t} \mathrm{ha}^{-1}\right)$, por meio da aferição de suas massas em balança eletrônica digital. As amostras foram desintegradas e recolheu-se individualmente, em frascos estéreis, o caldo extraído para a condução das aferições qualitativas, as quais foram conduzidas conforme orientações do Conselho dos
Produtores de Cana, Açúcar e Álcool do Estado de São Paulo (CONSECANA-SP, 2003).

Os resultados relacionados com o modo de aplicação da torta de filtro foram submetidos à análise de variância e teste de médias (Tukey) a 5\% de significância e, para avaliação dos dados obtidos relacionados com as doses de torta de filtro aplicadas, procedeu-se a análise de regressão polinomial. Todas as análises foram realizadas com o auxilio do software SANEST ${ }^{\circledR}$.

\section{RESULTADOS E DISCUSSÃO}

$\mathrm{Na}$ avaliação dos efeitos da aplicação da torta de filtro na linha superficial e na entrelinha incorporada ao solo sobre as variáveis BRIX, POL, TCH e TPH, os resultados mostram que os efeitos sobre estas variáveis revelaram-se estatisticamente iguais para os dois modos de aplicação da torta de filtro (Tabela 4). Esses resultados concordam com os obtidos por Tasso Júnior et al. (2000) em estudos sobre a produtividade e qualidade de cana-deaçúcar cultivada em solo tratado com adubação orgânica a base de lodo de esgoto, quando observaram que o modo de aplicação do resíduo (na linha ou na entrelinha da cana) não interferiu nas características quantitativas e qualitativas da soqueira.

Os diferentes modos de aplicação da torta de filtro não apresentaram diferença significativa sobre as variáveis em estudo. Em função da imobilidade do fósforo, a localização do adubo fosfatado influencia na absorção pela planta. Schroo (1956) e Kovar \& Barbeer (1989) observaram que é importante aumentar o teor desse elemento ao redor do sistema radicular das plantas e Nunes Júnior (2005) afirma que a matéria orgânica da torta de filtro, mesmo quando aplicada na entrelinha, reduz a fixação do fósforo pelos óxidos de ferro e alumínio, disponibilizando esse elemento às raízes. Além disso, a reação da matéria orgânica da torta, por permitir maior estabilidade de agregados, potencializa a absorção de nutrientes. Os resultados observados nesse trabalho foram antagônicos às considerações citadas, pois os resultados obtidos pela incorporação da torta de filtro aplicada na entrelinha igualaram-se à aplicação do resíduo na linha superficial, sendo então não significativos. 
Tabela 3 - Quantidade de nutrientes em função dos tratamentos.

\begin{tabular}{cccccccc}
\hline Adubação & $\mathrm{N}$ & $\mathrm{P}_{2} \mathrm{O}_{5}$ & $\mathrm{~K}_{2} \mathrm{O}$ & $\begin{array}{c}\mathrm{Ca} \\
\mathrm{kg} \mathrm{ha}^{-1}\end{array}$ & $\mathrm{Mg}$ & $\mathrm{S}$ & M.O. \\
\hline Adubação mineral da usina & 50 & 35 & 120 & 13,14 & - & 7,5 & - \\
$10 \mathrm{t} \mathrm{ha}^{-1}$ de torta de filtro & 54,34 & 26,31 & 8,58 & 69,50 & 7,44 & 11,15 & 574,86 \\
$20 \mathrm{t} \mathrm{ha}^{-1}$ de torta de filtro & 108,68 & 52,62 & 17,16 & 139 & 14,88 & 22,30 & 1149,72 \\
$40 \mathrm{t} \mathrm{ha}^{-1}$ de torta de filtro & 217,36 & 105,24 & 34,32 & 278 & 29,76 & 44,60 & 2299,44 \\
$80 \mathrm{t} \mathrm{ha}^{-1}$ de torta de filtro & 434,72 & 210,48 & 68,64 & 556 & 59,52 & 89,20 & 4598,88 \\
\hline
\end{tabular}

Tabela 4 - Efeito dos modos de aplicação da torta de filtro sobre variáveis tecnológicas e de produtividade da cana-deaçúcar; Goianésia/GO, 2004.

\begin{tabular}{ccccc}
\hline $\begin{array}{c}\text { Modo de aplicação da } \\
\text { torta de filtro }\end{array}$ & $\begin{array}{c}\text { BRIX médio } \\
(\%)\end{array}$ & $\begin{array}{c}\text { POL médio } \\
(\%)\end{array}$ & $\begin{array}{c}\text { TCH médio } \\
\left(\mathrm{t} \mathrm{ha}^{-1}\right)\end{array}$ & $\begin{array}{c}\text { TPH médio } \\
\left(\mathrm{t} \mathrm{ha}^{-1}\right)\end{array}$ \\
\hline Na linha superficial & $22,55 \mathrm{a}$ & $16,78 \mathrm{a}$ & $89,74 \mathrm{a}$ & $15,19 \mathrm{a}$ \\
Na entrelinha incorporada & $22,86 \mathrm{a}$ & $16,99 \mathrm{a}$ & $89,79 \mathrm{a}$ & $15,02 \mathrm{a}$ \\
\hline CV\% & 3,091 & 2,61 & 10,24 & 9,993 \\
\hline
\end{tabular}

Médias, em mesma coluna, seguidas de mesma letra, não diferem entre si pelo teste de Tukey a 5\%.

\section{Efeito das doses da torta de filtro sobre as variáveis}

Foram consideradas as doses de 10, 20, 40 e $80 \mathrm{t} \mathrm{ha}^{-1}$ de torta de filtro e a testemunha absoluta.

As variáveis que apresentaram ganho com o aumento das doses de torta de filtro foram: TCH (Figura 1) e TPH (Figura 2).

Observou-se que a aplicação da torta de filtro incrementou com ajuste quadrático na produtividade de colmos (Figura 1) e na produtividade de sacarose (Figura 2), atingindo ponto de máximo na dose de 57,59 e 56,90 tha-1 de torta de filtro, respectivamente.

As variáveis BRIX (Figura 3) e POL (Figura 4) apresentaram queda em seus valores com o aumento das doses de torta de filtro aplicadas.

Na Figura 3, mostra-se que o BRIX no caldo da canade-açúcar apresentou resposta linear para as diferentes doses de torta de filtro aplicadas, com inclinação da reta negativa indicando uma relação inversamente proporcional entre Brix do caldo da cana e dose de torta de filtro aplicada.

$\mathrm{Na}$ Figura 4, mostra-se que o polinômio de regressão, para avaliar os efeitos das diferentes doses de torta de filtro aplicadas sobre a variável POL\% da cana, resultou em uma equação quadrática.

A aplicação de torta de filtro proporcionou ganho na produtividade de colmos $(\mathrm{TCH})$ da soqueira $\left(\mathrm{t} \mathrm{ha}^{-1}\right)$ e mesmo com a queda na qualidade tecnológica da cana, medida pelo BRIX \% (Figura 3) e POL \% (Figura 4), houve aumento da produtividade sacarose (TPH) por área $\left(\mathrm{t} \mathrm{ha}^{-1}\right)$ (Figura 2). Os ganhos observados podem também ser explicados pela alteração da fertilidade do solo proporcionada pela torta de filtro, que proporciona uma nutrição mais adequada da canade-açúcar. Os efeitos das diferentes doses de torta de filtro sobre as variáveis TCH, TPH, BRIX e POL, podem ser atribuídos, em grande parte, aos benefícios proporcionados pela matéria orgânica da torta de filtro e pelos nutrientes nela encontrados, destacando-se o fósforo e nitrogênio. Resultados semelhantes foram observados por Tasso Júnior (2007), sendo a produtividade de colmo e de açúcar foi maior quando utilizados os resíduos, porém, complementados com fontes minerais.

Como os resultados das análises estatísticas da POL\% constatou-se que houve diminuição de POL\% com o aumento das doses de torta de filtro aplicadas. Infere-se que as variações observadas nas produções de açúcar (TPH) foram consequências do aumento da produção agrícola (TCH).

Efeito do modo de aplicação da torta de filtro sobre as variáveis

Os resultados encontrados mostram que não houve diferença significativa para a interação entre doses de torta de filtro e os modos de aplicação, para nenhuma das variáveis avaliadas. Portanto, a dose de torta de filtro que favorece o aumento das variáveis TCH e TPH, BRIX e POL, não depende do modo de aplicação da torta de filtro. No entanto, como não houve diferença entre os modos de aplicação, provavelmente, uma opção mais econômica seria na linha superficial, pois não haveria necessidade de incorporação. 


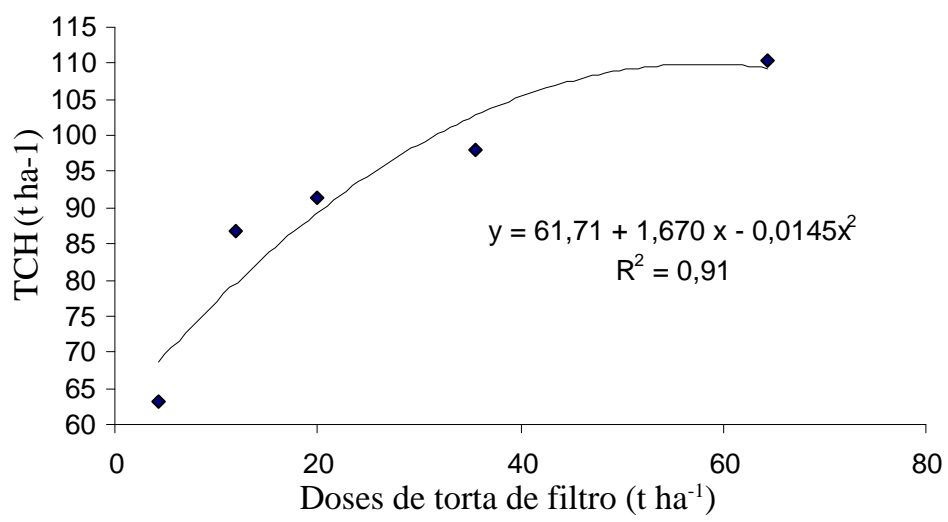

Figura 1 - Produtividade média de colmos de cana-de-açúcar (TCH) em função das doses de torta de filtro aplicadas; Goianésia/GO, 2004.

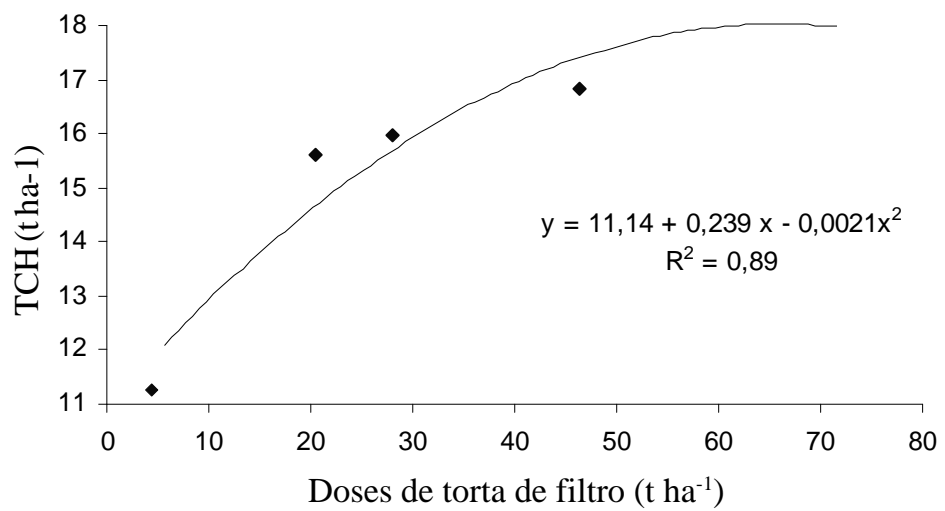

Figura 2 - Produtividade média de sacarose na cana-de-açúcar (TPH) em função das doses de torta de filtro aplicadas; Goianésia/GO, 2004.

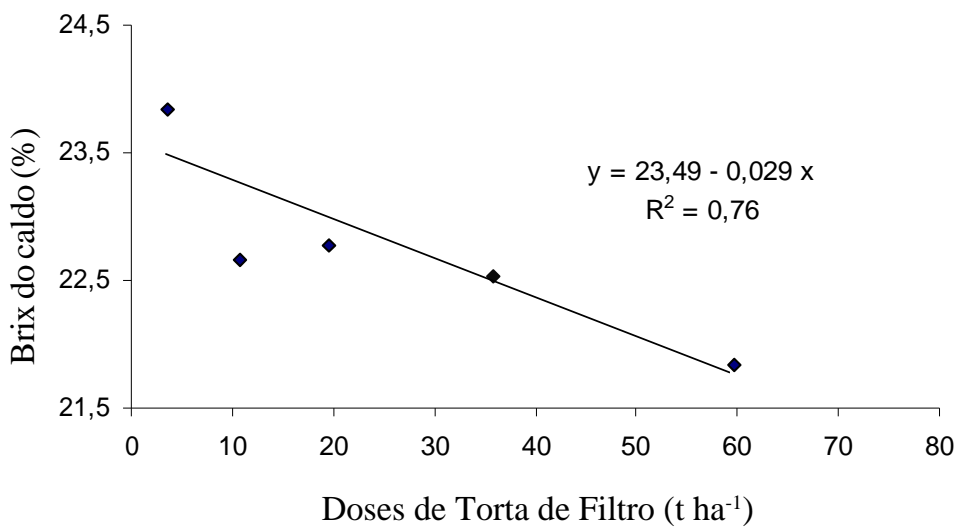

Figura 3 - Teor médio de sólidos totais do caldo da cana-de-açúcar (BRIX\%) em função das diferentes doses de torta de filtro aplicadas; Goianésia/GO, 2004. 


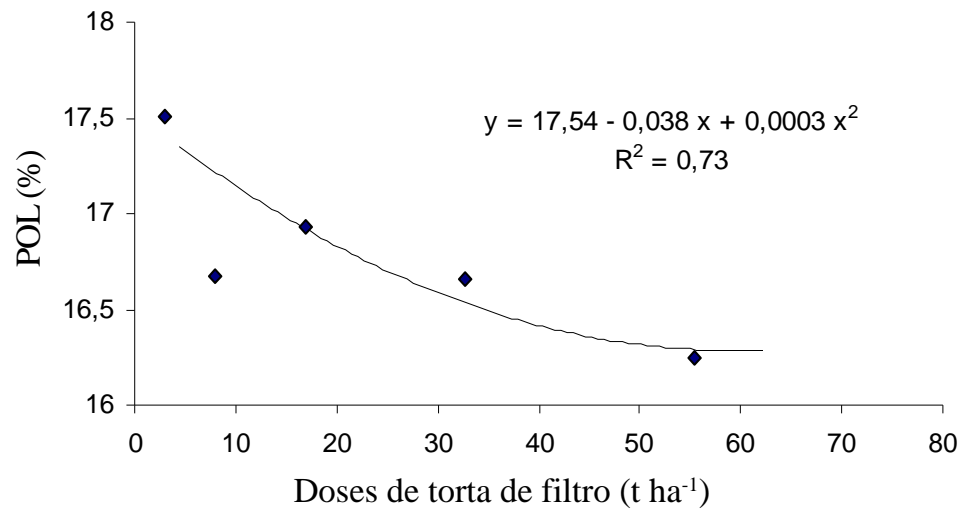

Figura 4 - Teor médio de sacarose da cana-de-açúcar (POL\%) em função das diferentes doses de torta de filtro aplicadas; Goianésia/GO, 2004.

\section{CONCLUSÕES}

A aplicação de torta de filtro diminuiu o Brix do caldo e o Pol da cana, entretanto, houve aumento na produtividade de colmos por hectare $(\mathrm{TCH})$ e na produtividade de sacarose por hectare (TPH);

Os modos de aplicação (linha e entrelinha) da torta de filtro não influenciaram a qualidade tecnológica da cana e a produtividade de colmos.

\section{AGRADECIMENTOS}

Ao Dr. Ricardo Fontoura Siqueira e aos técnicos da Usina Jalles Machado (Goianésia - GO) pelo auxílio na instalação e condução do experimento e ao $\mathrm{CNPq}$ e FAPEMIG pelo suporte financeiro.

\section{REFERÊNCIAS BIBLIOGRÁFICAS}

ALVARENGA, R.P.; QUEIROZ, T.R. Caracterização dos aspectos e impactos econômicos, sociais e ambientais do setor sucroalcooleiro Paulista. In: CONGRESSO DA SOCIEDADE BRASILEIRA DE ECONOMIA, ADMINISTRAÇÃO E SOCIOLOGIA RURAL, 46., 2008, Rio Branco. Anais... Rio Branco, 2008. p.21.

ANJOS, I.A.; ANDRADE, L.A.B.; GARCIA, J.C.; FIGUEIREDO, P.A.M.; CARVALHO, G.J. Efeitos da adubação orgânica e da época de colheita na qualidade da matéria-prima e nos rendimentos agrícola e de açúcar mascavo artesanal de duas cultivares de cana-de-açúcar (cana-planta). Ciência e Agrotecnologia, Lavras, v.31, n.1, p.59-63, jan./fev. 2007.

BARBOSA, M.H.P.; OLIVEIRA, M.W.; DAMASCENO, C.M.; MENDES, L.C. Acúmulo e alocação de nutrientes pela RB72454 no ciclo da cana-planta. In. CONGRESSO NACIONAL DA SOCIEDADE DOS TÉCNICOS AÇÚCAREIROS E ALCOOLEIROS DO BRASIL, 8., 2002, Recife. Anais... Recife: STAB, 2002. p.264-267.

BATAGLIA, O.C.; FURLANI, A.M.C.; TEIXEIRA, J.P.F.; FURLANI, P.R.; GALLO, J.R. Métodos de análise química de plantas. Campinas: Instituto Agronômico, 1985. 48p. (Boletim técnico, 78).

BITTENCOURT, V.C.; PAIXÃO, A.C.S.; CESARIN, L.G.; SOUZA, S.R. Cana conquista o Mato Grosso do Sul.

IDEA News, Ribeirão Preto, n.63, jan. 2006. Disponível em: 〈http:///www.ideaonline.com.br/s. Acesso em: $10 \mathrm{dez}$. 2006.

CERRI, C.C.; POLO, A.; ANDREUX, F.; LOBO, M.C.; EDUARDO, B.P. Resíduos orgânicos da agroindústria canavieira: 1 ., características físicas e químicas. STAB: Açúcar, Álcool e Subprodutos, Piracicaba, v.6, n.1, p.3437, 1988.

CONSELHO DOS PRODUTORES DE CANA, AÇÚCAR E ÁLCOOL DO ESTADO DE SÃO PAULO. Normas de avaliação da qualidade da cana-se-açúcar: a qualidade da cana fornecida às unidades industriais. São Paulo, 2003. Disponível em: <http://www.unica.com.br/files/ iconsecana/normaseprecos.pdf 2003 .

KORNDÖRFER, G.H. Resposta da cultura da cana-deaçúcar à adubação fosfatada. Informações

Agronômicas, Piracicaba, v.102, p.7, jun. 2003. 
KOVAR, J.L.; BARBER, S.A. Reasons for differences among soils in placement of phosphorus for maximum predicted uptake. Soil Science Society American Journal, Madison, v.53, p.1733-1736, 1989.

MEDEIROS, S.C.L. Efeito da adubação fosfatada em plantio e em soqueiras de cana-de-açúcar. [S.l.: s.n.], 1988.

MEDEIROS, S.C.L.; RIBEIRO, S.R.; CONEGLIAN, C.M.R. Impactos da agroindústria canavieira sobre o meio ambiente. In: FÓRUM DE ESTUDOS CONTÁBEIS, 3., 2003, Limeira. Anais... Limeira, 2003. p.7.

NUNES JÚNIOR, D. O insumo torta de filtro. IDEA News, Ribeirão Preto, 2005.

NUNES JÚNIOR, D. A redução da adubação e a produtividade. STAB, Piracicaba, v.17, n.3, p.16, 1999.

OLIVEIRA, F.C.; MATTIAZZO, M.E.; MARCIANO, C.R.; ROSSETO, R. Efeitos de aplicações sucessivas de lodo de esgoto em Latossolo Amarelo distrófico cultivado com cana-de-açúcar: carbono orgânico, condutividade elétrica, pH e CTC. Revista Brasileira de Ciência do Solo, Viçosa, v.26, p.505-519, 2002.

PRODUÇÃO de cana de açúcar deve bater recordes na safra 2005-2006. UOL Economia, São Paulo, 4 jan. 2006. Disponível em: 〈http://noticias.uol.com.br/economia/, rultnot/efe/2006/01/04/ult1767u58086.jhtm $>$. Acesso em: 8 dez. 2006 .

SCHROO, H. The efficient use of phosphate fertilizers on acid phosphate fixing sugarcane soils. Society Sugar Cane Technology, New Delhi, n.1, p.497-503, 1956.

SLEIGHT, D.M.; SANDER, D.H.; PETERSON, G.A. Effect of fertilizer of phosphorus. placement on the availability of phosphorus. Soil Science Society American Journal, Madison, v.48, p.336-340, 1984.

ZAMBELLO JUNIOR, E.; HAAG, H.P.; ORLANIDO

FILHO, J. Adubação NPK e localização do fertilizante em soqueiras de cana-de-açúcar, variedade CB 41-76. Brasil Açucareiro, Rio de Janeiro, v.96, n.4, p.34-46, out. 1980. 